\title{
Laboratory test utilization: an opportunity for laboratory empowerment
}

\begin{abstract}
Shifts in the global healthcare climate is causing clinical laboratories to reexamine how the laboratory operates, particularly when it comes to test ordering. Laboratories are focusing on decreasing unnecessary testing not solely to save costs, but I an effort to improve patient care, outcomes and satisfaction. Unnecessary testing also has the potential to create patient safety issues. The higher volume of unwarranted testing performed, the greater the potential for error. Particularly since any test with a specificity or sensitivity less than $100 \%$ has the potential for false positives and negatives. Laboratories are beginning to focus on this overutilization of testing, and in doing so are finding their place at the table with clinicians and other peers.
\end{abstract}

Volume I Issue I - 2015

\author{
Lee B Springer \\ Colorado Technical University, USA
}

Correspondence: Lee B Springer Colorado Technical University, 4435 N Chestnut St Colorado Springs CO, 80907, Tel6129|69732, Email navymedtech@hotmail.com

Received: July 23, 2015 | Published: July 27, 2015

\section{Introduction}

Over the past few decades the clinical laboratory has become vastly more robust and dynamic. Previously clinicians had at most a few dozen standard tests that could be ordered, with very little specialized testing available. This has changed drastically over the past decade, with many hospital laboratories offering a bevy of standard and specialized testing. The logic behind the ever increasing test menu was often based upon financial data such as the volume of specific tests being sent out, reimbursement rates, and market competition differentiators. These are all prime business reasons for expanding a testing menu, particularly since laboratory testing provides the most cost effective, least invasive source for objective information for making clinical decisions. However, this approach often lacks clinical reasoning. Where the problem lies is differentiating between the utility, necessity and application of a test, similar to what pharmacies do when establishing their formulary. The pharmacy serves as a valuable blueprint that the laboratory community can follow for test utilization.

Pharmacists work in conjunction with physicians, employing evidence based practice and their expert knowledge to establish formularies, dosages, and develop clinical protocols clinical protocols. The clinical laboratory is the exact opposite, our focus has been providing the test as quickly and consistently as possible, and for the most part regardless of reason or clinical picture. While this is well suited to the acute care environment, it is not conducive to population health management, and primary care. This approach is considerably more ineffective with the increasing number of specialized genetic and molecular tests available through commercial reference laboratories. The reason for this is simple, physicians simply don't fully understand what it is they are ordering, or how the information can be utilized in clinical care. This in turn escalates the cost of care to the patient and diminishes the level of care provided, and in many cases can be costly to both the patient and the hospital. Progressive laboratories have poised themselves to address this problem as an opportunity to correct inefficiencies and not as restricting clinician decisions regarding testing.

Many of these laboratories are establishing testing formularies and managing them in the same respects that a pharmacy manages theirs, with official processes and multi-disciplinary review teams to review any changes or recommendations. This creates a setting where it is no longer about restricting choice, but establishing evidence based practice for specific testing. Additionally, laboratory administrators with the help of pathologist and clinicians are developing testing algorithms to ensure that the proper cascade of test ordering. These algorithms serve four key purposes, the first is to ensure that the entry level screening test is performed prior to more complicated diagnostic testing, second to reduce the volume of inappropriately ordered tests, third to enhance the clinical relevance of the information gained from the testing, and the forth is to reduce costs.

Establishing laboratory test utilization programs is a daunting task, and one that requires not only engaging key stakeholders, but may also involve the utilization of technology. Optimizing electronic medical records to support and direct laboratory test management is key not only for its success, but ensuring that it remains efficient and integrated within the flow of clinical care. Each lab will need to develop their own processes and unique combination of strategies in order to effectively establish and maintain a system for test utilization. The benefit of establishing these programs is the enhanced collaboration between clinicians and laboratorians, reduction in costs, improvement in clinical care, and the overall positive impact for the patients themselves.

\section{Acknowledgements}

None.

\section{Conflict of interest}

The author declares no conflict of interest. 